\title{
AN ELEMENTARY ESTIMATE FOR THE $k$-FREE INTEGERS ${ }^{1}$
}

\author{
BY ECKFORD COHEN
}

Communicated by George Whaples, December 24, 1962

1. Introduction. In this note $k$ will denote a fixed integer $>1$. Let $Q_{k}$ denote the sequence of $k$-free integers, that is, the integers whose prime factors are all of multiplicity $<k$. Also, let $\zeta(k)$ denote the sum of the series, $\sum_{n=1}^{\infty} n^{-k}$.

In this paper we prove the identity,

$$
\sum_{r=1}^{\infty}\left(\frac{\mu(r)}{J_{k}(r)}\right) c_{k}(n, r)= \begin{cases}\zeta(k) & \text { if } n \in Q_{k}, \\ 0 & \text { if } n \in Q_{k},\end{cases}
$$

where $\mu(r)$ is the inversion function of number theory, $J_{k}(r)$ the Jordan totient of order $k$, and $c_{k}(n, r)$ is the generalized Ramanujan sum defined by (3) below. This is a special case of a much more general result proved in [4, Theorem 6]. In view of the intrinsic interest of the relation (1), an independent proof of its validity seems justified.

As a consequence of (1), we prove, without resorting to remainder estimates of series, the following asymptotic formula for the number $Q_{k}(x)$ of integers of $Q_{k}$ not exceeding $x$ :

$$
Q_{k}(x)=x / \zeta(k)+O\left(x^{1 / k+c}\right),
$$

for all $\epsilon>0$ (see Remark $2, \S 3$ ). If one assumes an estimate for the remainder of the series $\sum_{n=1}^{\infty} n^{-s}, s>1,(2)$ can be shown easily to hold with $\epsilon=0$ (see for example, $[7, \S 18.6 ; 3, \S 2]$ ).

The method employed in the proof of (2) is essentially a generalization and refinement of a method introduced by Carmichael [1]. Carmichael obtained approximations for the average order of certain arithmetical functions using Ramanujan's trigonometric series expansions, in connection with an estimate involving Ramanujan's sum, $c(n, r)=c_{1}(n, r)$. The present discussion employs the more general sum $c_{k}(n, r)$ introduced by the author [2] and an appraisal for $c_{k}(n, r)$ which is sharper than the corresponding estimate of Carmichael (see (9) below).

2. Proof of (1). The function $c_{k}(n, r)$ is defined by

$$
c_{k}(n, r)=\sum_{a(\bmod r) ;\left(a, r^{k}\right)_{k}=1} \exp \left(2 \pi i a n / r^{k}\right),
$$

${ }^{1}$ This research was supported in part by the National Science Foundation grant G-19012. 
where the summation is over all $a(\bmod r)$, whose greatest common $k$ th power divisor with $r^{k}$ is 1 . The expansion (2) can be obtained most readily by applying the arithmetical evaluation of (3), [2, (2.5)],

$$
c_{k}(n, r)=\sum_{d\left|r, d^{k}\right| n} d^{k} \mu(r / d)
$$

Let $\sigma_{s}(n)$ denote the sum of the sth powers of the divisors of $n$, $\sigma_{1}(n) \equiv \sigma(n)$. It is noted that the series in (2) converges absolutely for each $n$, by virtue of the estimate, $\left|c_{k}(n, r)\right| \leqq \sigma(n)$ (deducible from (4)), and the simple property,

$$
J_{k}(n) \asymp n^{k} \text { as } n \rightarrow \infty \text {, }
$$

which is a consequence of the evaluation, $J_{k}(r) / r^{k}=\sum_{d \mid r} \mu(d) / d^{k}$.

Denoting the series in (2) by $\Sigma$, we have

$$
\Sigma=\sum_{r=1}^{\infty} \frac{\mu(r)}{J_{k}(r)} \sum_{d \delta=r ; d^{k} \mid n} d^{k} \mu(\delta)=\sum_{d^{k} \mid n} \frac{d^{k} \mu(d)}{J_{k}(d)} \sum_{\delta=1 ;}^{\infty} \frac{\mu^{2}(\delta)}{J_{k}(\delta)},
$$

by the multiplicative properties of $\mu(r)$ and $J_{k}(r)$. Letting $p$ designate primes, we have the Euler product factorization,

$$
\sum_{n=1 ;(n, r)=1}^{\infty} \frac{\mu^{2}(n)}{J_{k}(n)}=\frac{\prod_{p}\left(1+\left(p^{k}-1\right)^{-1}\right)}{\prod_{p \mid r}\left(1+\left(p^{k}-1\right)^{-1}\right)}=\frac{\zeta(k)}{r^{k} / J_{k}(r)} .
$$

Combination of (6) and (7) yields then (cf. $[5, \$ 17.8]$ )

$$
\sum_{r=1}^{\infty}\left(\frac{\mu(r)}{J_{k}(r)}\right) c_{k}(n, r)=\zeta(k) \sum_{d^{k} \mid n} \mu(d)=q_{k}(n) \zeta(k),
$$

where $q_{k}(n)$ denotes the characteristic function of $Q_{k}$. This completes the proof of (1).

3. Proof of (2). The following estimate for $c_{k}(n, r)$ is basic for our discussion.

LEMma. If $0<\alpha<1$, then for all $\epsilon>0$,

$$
\sum_{n \leq x} c_{k}(n, r)= \begin{cases}x+O\left(x^{\alpha}\right) & \text { if } r=1, \\ O\left(x^{\alpha} r^{k(1-\alpha)+\epsilon)}\right. & \text { if } r>1,\end{cases}
$$

uniformly in $r$.

REMARK 1. This result and its proof are also valid in case $k=1$. 
Proof. If $[x]$ denotes the largest integer $\leqq x$, then evidently $[x]=x+O\left(x^{\alpha}\right)$ for $x>0$; hence by $(4)$,

$$
\begin{aligned}
\sum_{n \leqq x} c_{k}(n, r) & =\sum_{n \leqq x} \sum_{d e=r ; d k \delta=n} d^{k} \mu(e)=\sum_{d e=r} d^{k} \mu(e)\left[\frac{x}{d^{k}}\right] \\
& =\sum_{d e=r} d^{k} \mu(e)\left\{\frac{x}{d^{k}}+O\left(\frac{x^{\alpha}}{d^{k \alpha}}\right)\right\} \\
& =x \sum_{d e=r} \mu(e)+O\left(x^{\alpha} \sigma_{k(1-\alpha)}(r)\right) .
\end{aligned}
$$

But it is a known elementary fact that $\sigma_{s}(r)=O\left(r^{s+c}\right)$ if $s>0$ (see Remark 2). Thus the lemma results by the characteristic property of $\mu(n)$.

To estimate $Q_{k}(x)$, we have by (1) and (9),

$$
\begin{aligned}
Q_{k}(x) & =\sum_{n \leqq x} q_{k}(n)=\frac{1}{\zeta(k)} \sum_{r=1}^{\infty}\left(\frac{\mu(r)}{J_{k}(r)}\right) \sum_{n \leqq x} c_{k}(n, r) \\
& =\frac{1}{\zeta(k)}\left(x+O\left(x^{\alpha}\right)\right)+O\left(x^{\alpha} \sum_{r=2}^{\infty} \frac{r^{k(1-\alpha)+\epsilon}}{J_{k}(r)}\right),
\end{aligned}
$$

where $0<\alpha<1$. In particular, with $\alpha=1 / k+\epsilon$, it follows by (5) that

$$
Q_{k}(x)=\frac{x}{\zeta(k)}+O\left(x^{1 / k+\epsilon}\right)+O\left(x^{1 / k+\epsilon} \sum_{r=2}^{\infty} \frac{1}{r^{1+\epsilon}}\right),
$$

from which one obtains (2) for all $\epsilon>0$.

REMARK 2. The property of the $J$-function used in $\$ 2$ and that of the $\sigma$-function used in this section can be deduced, assuming simply the convergence of $\sum 1 / n^{s}$ for $s>1$ (cf. $[7, \S 18.4]$ ).

ADDITIONAL REMARKS. Insofar as the method of this section makes no appeal to the calculus, either finite or infinitesimal, it can be described as essentially algebraic in nature. Additional applications of this method will be made in a longer paper to appear later [5].

To paraphrase Hardy [6], the formula (1) suggests "in a very striking way" a harmonic pattern in the behavior of the sequence $Q_{k}$. Hardy's comment related to Ramanujan's trigonometric series development of $\sigma(n)$. For a theory of "mean even" arithmetical functions, including both $\sigma(n)$ and $q_{k}(n)$, the reader is referred to the paper [4], which is to appear elsewhere. 


\section{BIBLIOGRAPHY}

1. R. D. Carmichael, On expansions of arithmetical functions, Proc. Nat. Acad. Sci. 16 (1930), 613-616.

2. Eckford Cohen, An extension of Ramanujan's sum, Duke Math. J. 16 (1949), $85-90$.

3. - Some sets of integers related to the k-free integers, Acta Sci. Math. Szeged 22 (1961), 223-233.

4. - On the mean parity of arithmetical functions, (to appear).

5. - Cauchy product estimates of arithmetical functions, (to appear).

6. G. H. Hardy, Ramanujan, Chelsea, New York (in particular, p. 141, paragraph 2).

7. G. H. Hardy and E. M. Wright, The theory of numbers, 3rd ed., Clarendon, Oxford, 1954.

The University of Tennessee 